December 2, 2018

\title{
Radiating sources in higher-dimensional gravity
}

\author{
Hongya Liu* \\ Department of Physics, Dalian University of Technology, \\ Dalian, 116024, P.R. China \\ Paul S. Wesson ${ }^{\dagger}$ \\ Department of Physics, University of Waterloo, \\ Waterloo, Ontario N2L 3G1, Canada
}

\begin{abstract}
We study a time-dependent 5D metric which contains a static $4 \mathrm{D}$ sub-metric whose 3D part is spherically symmetric. An expansion in the metric coefficient allow us to obtain close-to Schwarzschild approximation to a class of spherically-symmetric solutions. Using Campbell's embedding theorem and the induced-matter formalism we obtain two 4D solutions. One describes a source with the stiff equation of state believed to be applicable to dense astrophysical objects, and the other describes a spherical source with a radial heat flow.
\end{abstract}

PACS number(s): 04.20.Jb, $11.10 \mathrm{Kk}$

\footnotetext{
*Email: hyliu@dlut.edu.cn

†Email: wesson@astro.uwaterloo.ca
} 


\section{INTRODUCTION}

Campbell showed the theorem that any solution of the Einstein equations in $N$ dimensions can be locally embedded in a Ricci-flat manifold of $(N+1)$ dimensions whose field equations in terms of the Ricci tensor are $R_{A B}=0(A, B=0,1, \ldots, N) .{ }^{1}$ Tavakol and coworkers have recently noted the relevance of this to the embedding of lower-dimensional (possibly quantizeable) gravity in 4D Einstein gravity, ${ }^{2}$ and the technique is clearly applicable to the recovery of solutions of $4 \mathrm{D}$ general relativity from 10D superstrings, 11D supergravity and M-theory. ${ }^{3-6}$ A major application of Campbell's theorem is to induced-matter theory, wherein solutions of the 4D Einstein equations with matter are recovered from the 5D Kaluza-Klein equations in apparent vacuum. ${ }^{7}$ This approach has been applied to cosmology, ${ }^{8}$ clusters of galaxies ${ }^{9}$ and the solar system, ${ }^{10,11}$ where there is agreement with observational data. It is valuable in application to general relativity, since solutions of the Kaluza-Klein equations can yield new solutions of the Einstein equations. With regard to the latter, sphericallysymmetric sources such as stars can be modelled in the simplest way by the interior and exterior Schwarzschild solutions. But to include the radiation outside a star, more complicated solutions are required. ${ }^{12}$ These include the Vaidya metric which uses a retarded time coordinate to describe a radiating atmosphere, ${ }^{13}$ the metrics of Herrera and coworkers wherein spheres of matter are matched to exterior spacetimes, ${ }^{14-16}$ and the metrics of Glass and Krisch which extend the Vaidya solution to include both a radiation field and a string fluid. ${ }^{17}$ However, despite extensive work on starlike solutions of the 4D equations and the existence of Campbell's theorem which shows that such can be embedded in the ND equations, not much work has been done on spherically-symmetric solutions of the 5D equations. Notable exceptions are solutions which have an isothermal equation of state in $4 \mathrm{D}^{9}$ and ones which are flat in $5 \mathrm{D}$ but curved in $4 \mathrm{D} .{ }^{18}$ We will therefore present an analysis of a class of 5D metrics, and illustrate their relevance by isolating two 4D solutions. One describes a source with the stiff equation of state believed to be applicable to dense astrophysical objects, and the other describes a spherical source with radiation.

\section{5D METRICS WITH 4D SPHERICAL SOURCES}

In this section we let upper-case Latin indices run 0-4 and lower-case Greek indices run 0-3. We absorb the speed of light and the gravitational constant through the choices of units $c=1,8 \pi G=1$. The coordinates are $x^{A}=t, r, \theta, \phi, y$ with $d \Omega^{2} \equiv d \theta^{2}+\sin ^{2} \theta d \phi^{2}$ with (3D) spherical symmetry.

Consider the 5D line element with interval

$$
\begin{aligned}
d S^{2} & =g_{A B} d x^{A} d x^{B}=\Phi^{2} d s^{2}-\Phi^{-4} d y^{2} \\
d s^{2} & =g_{\alpha \beta} d x^{\alpha} d x^{\beta}
\end{aligned}
$$


where $g_{\alpha \beta}=g_{\alpha \beta}\left(x^{\mu}\right)$ and $\Phi=\Phi\left(x^{\mu}\right)$.. This metric is broad in scope and has been used in other contexts. ${ }^{19}$ It also has the advantage that the $5 \mathrm{D}$ field equations $R_{A B}=0$ break down neatly into a set involving the $4 \mathrm{D}$ Ricci tensor and a conservation-type equation for the scalar field $\left(g_{44}=-\Phi^{-4}\right)$ :

$$
\begin{aligned}
R_{\alpha \beta} & =6 \Phi^{-2} \Phi_{\alpha} \Phi_{\beta}, \\
\Phi \Phi_{; \alpha}^{\alpha}-\Phi^{\alpha} \Phi_{\alpha} & =0 .
\end{aligned}
$$

Here $\Phi^{\alpha}=g^{\alpha \beta} \Phi_{\beta}, \Phi_{; \alpha}^{\alpha}=g^{\alpha \beta} \Phi_{\alpha ; \beta}$ and $R_{\alpha \beta}$ in (3) is made of $g_{\alpha \beta}$. Equations (3) and (4) admit many different types of solution, but here we choose a time-dependent scalar field and a static $4 \mathrm{D}$ geometry:

$$
\begin{aligned}
\Phi & =\Phi(t), \\
d s^{2} & =B(r) d t^{2}-A(r) d r^{2}-r^{2} d \Omega^{2} .
\end{aligned}
$$

These require that the LHS of (3) should be independent of $t$ and the RHS of (3) should be independent of $r$. Therefore both sides should equal to a constant, say, $6 \lambda^{2}$. So we must have

$$
\Phi=e^{\lambda t} .
$$

Substituting this into equation (4), we find that (4) is satisfied. Using (6) and (7) in (3) gives the non-vanishing components

$$
\begin{aligned}
& R_{00}=\frac{B^{\prime \prime}}{2 A}-\frac{B^{\prime}}{4 A}\left(\frac{A^{\prime}}{A}+\frac{B^{\prime}}{B}\right)+\frac{1}{r} \frac{B^{\prime}}{A}=6 \lambda^{2}, \\
& R_{11}=-\frac{B^{\prime \prime}}{2 B}+\frac{B^{\prime}}{4 B}\left(\frac{A^{\prime}}{A}+\frac{B^{\prime}}{B}\right)+\frac{1}{r} \frac{A^{\prime}}{A}=0, \\
& R_{22}=\sin ^{-2} \theta R_{33}=1-\frac{1}{A}+\frac{r}{2 A}\left(\frac{A^{\prime}}{A}-\frac{B^{\prime}}{B}\right)=0,
\end{aligned}
$$

with $A^{\prime} \equiv d A / d r$. These are three equations in two unknowns, and determine a class of solutions which is time-dependent in $5 \mathrm{D}$ via (7) but static in $4 \mathrm{D}$ and spherically symmetric in $3 \mathrm{D}$.

Let us manipulate (8)-(10). The sum of $A B^{-1} R_{00}$ of (8) and $R_{11}$ of (9) gives

$$
\frac{A^{\prime}}{A}+\frac{B^{\prime}}{B}=6 \lambda^{2} r \frac{A}{B}
$$

And we can rewrite (10) as

$$
\frac{A^{\prime}}{A}-\frac{B^{\prime}}{B}=-\frac{2}{r}(A-1)
$$


Using (11) and (12), we verify that (8) and (9) are satisfied. Therefore, we need to solve (11) and (12) for $A$ and $B$. Now (12) can be written as $(d / d r) \ln (A / B)=$ $-2(A-1) / r$. Integrating this we get

$$
B=A \exp \left[2 \int_{r_{0}}^{r} \frac{A-1}{r} d r\right] .
$$

Substituting this into (11), we obtain

$$
\frac{A^{\prime}}{A}+\frac{A-1}{r}=3 \lambda^{2} r \exp \left[-2 \int_{r_{0}}^{r} \frac{A-1}{r} d r\right] .
$$

Without loss of generality, let us introduce a mass function ${ }^{20,21}$ via

$$
A=\left(1-\frac{2 \mu(r)}{r}\right)^{-1}
$$

Then (14) becomes

$$
\mu^{\prime}=\frac{3}{2} \lambda^{2} r(r-2 \mu) \exp \left[2 \int_{r_{0}}^{r}\left(\frac{1}{r}-\frac{1}{r-2 \mu}\right) d r\right] .
$$

Here $r_{0}$ is a fiducial radius that can be chosen as appropriate to a physical situation. Thus if $r_{0} \rightarrow \infty$ we expect to recover the Schwarzschild case. The latter is indeed recovered for $\lambda=0$, when $\mu=M=$ constant and (13) reads

$$
B=A \exp \left[2 \int_{\infty}^{r} \frac{A-1}{r} d r\right]=1-\frac{2 M}{r} .
$$

That is, $\lambda=0$ specifies the Schwarzschild limit of a class of solutions determined by (16) and one or the other of (11) and (12).

\section{CLOSE-TO-SCHWARZSCHILD APPROXIMATION}

We can study close-to-Schwarzschild approximation by expanding the mass function $\mu(r)$ of (15) for $|\lambda|$ small (i.e. $\left.|\lambda|^{-1} \gg M\right)$. Thus we write

$$
\mu(r)=\sum_{n=0}^{\infty} \mu_{n}(r)
$$

with $\mu_{0}=M$. Here $\mu_{0}$ is the zero-order approximation of $\mu,\left(\mu_{0}+\mu_{1}\right)$ is the first-order approximation, and so on. To obtain $\mu_{1}$ we substitute $\mu_{0}$ for $\mu$ in the RHS of (16) to obtain $\mu_{1}^{\prime}$. Integrating $\mu_{1}^{\prime}$ gives $\mu_{1}$. Then we substitute $\left(\mu_{0}+\mu_{1}\right)$ for $\mu$ in the 
RHS of (16) to obtain $\mu_{2}^{\prime}$. In this way we obtain the following recursion formulae for evaluating $\mu_{n}(r)$ :

$$
\begin{aligned}
\mu_{0}^{\prime}= & 0 \\
\mu_{1}^{\prime}= & -\mu_{0}^{\prime}+\frac{3}{2} \lambda^{2} r(r-2 M) \exp \left[2 \int_{r_{0}}^{r}\left(\frac{1}{r}-\frac{1}{r-2 M}\right) d r\right] \\
& \cdots \ldots \ldots \ldots \ldots . . \\
\mu_{n}^{\prime}= & -\sum_{m=0}^{n-1} \mu_{m}^{\prime}+\frac{3}{2} \lambda^{2} r\left(r-2 \sum_{m=0}^{n-1} \mu_{m}\right) . \\
& \exp \left\{2 \int_{r_{0}}^{r}\left[\frac{1}{r}-\left(r-2 \sum_{m=0}^{n-1} \mu_{m}\right)^{-1}\right] d r\right\} .
\end{aligned}
$$

From this we find

$$
\mu_{1}^{\prime}=\frac{3}{2} \lambda^{2}\left(1-\frac{2 M}{r_{0}}\right)^{2} \frac{r^{3}}{r-2 M}
$$

and so

$$
\mu_{1}=\frac{3}{2} \lambda^{2}\left(1-\frac{2 M}{r_{0}}\right)^{2} \int_{r_{0}}^{r} \frac{r^{3} d r}{r-2 M} .
$$

In the region $r \gg 2 M$ and $r_{0} \gg 2 M$, we find

$$
\mu_{1} \approx \frac{1}{2} \lambda^{2} r^{3}
$$

where we have absorbed a constant term in $\mu_{0}$ without loss of generality, so the first-order approximation of $A(r)$ in (15) is

$$
A \approx\left(1-\frac{2 M}{r}-\lambda^{2} r^{2}\right)^{-1}
$$

Substituting this equation into (13), keeping only terms up to first order in $\lambda^{2}$ as well as in $M$, and neglecting a constant factor in $B$, we find

$$
B \approx 1-\frac{2 M}{r}+2 \lambda^{2} r^{2}
$$

From this coefficient and that of (23) we obtain the first order close-to-Schwarzschild approximation of the 5D solution as

$$
d S^{2} \approx e^{2 \lambda t}\left[\left(1-\frac{2 M}{r}+2 \lambda^{2} r^{2}\right) d t^{2}-\left(1-\frac{2 M}{r}-\lambda^{2} r^{2}\right)^{-1} d r^{2}-r^{2} d \Omega^{2}\right]-e^{-4 \lambda t} d y^{2}
$$


This metric is time-dependent. When $\lambda \rightarrow 0$, it tends to the 5D Schwarzschild solution. So we call (25) the close-to-Schwarzschild approximation for small $|\lambda|$, implying that the time-variation of the field should be very slow. We also find that the solution (25) belongs to the Type D class of the general time-dependent 5D metrics. ${ }^{22}$ As regards the $4 \mathrm{D}$ part inside the square bracket in (25), it is interesting to note that it does not define the 4D Schwarzschild-de Sitter solution. In the latter, $A$ and $B$ both contain a term $\Lambda r^{2} / 3$ where $\Lambda$ is the cosmological constant. By contrast, whereas $\lambda^{2}$ has the same physical dimensions as $\Lambda$ (namely time $^{-2}$ or length ${ }^{-2}$ ), $A$ and $B$ in (23) and (24) contain terms with different signs and different sizes. This situation is analogous to another in Kaluza-Klein theory, where the exact solution of the 5D field equations for a charged point mass does not exactly reproduce the 4D Reissner-Nordstrom solution. ${ }^{23}$ Since the 5D equations are richer than the 4D ones, such situations may be expected; but even so, (25) defines a new solution.

\section{TWO EXACT 4D SOLUTIONS}

There are, of course, many other solutions than those which are close to Schwarzschild. In general, we have a class of solutions of $R_{A B}=0$ with a metric

$$
d S^{2}=e^{2 \lambda t}\left[B d t^{2}-A d r^{2}-r^{2} d \Omega^{2}\right]-e^{-4 \lambda t} d y^{2}
$$

whose spacetime potentials $A, B$ are determined by (11),(12). Alternatively, they are determined by (16) and one or the other of (11), (12). We should recall, however, that while (8), (9), (10) are 4D relations and can be used as such, the class of metrics (26) is $5 \mathrm{D}$ in nature. This means that we can use Campbell's theorem ${ }^{1}$ and the induced-matter formalism ${ }^{7}$ to generate $4 \mathrm{D}$ solutions of Einstein's equations with their appropriate matter. We now proceed to show how the 5D metric (26) produces two exact $4 \mathrm{D}$ solutions.

\section{A. SOLUTION WITH A STIFF FLUID}

The first is obtained by splitting off the part inside square brackets in (26). The $4 \mathrm{D}$ metric is then

$$
d s^{2}=g_{\alpha \beta} d x^{\alpha} d x^{\beta}=B d t^{2}-A d r^{2}-r^{2} d \Omega^{2},
$$

and is static. We therefore expect that the source necessary to balance Einstein's equations will also be static. As mentioned above, the Kaluza-Klein equations $R_{A B}=$ $0(A, B=0,123,4)$ contain as a subset the Einstein equations $R_{\alpha \beta}-R g_{\alpha \beta} / 2=T_{\alpha \beta}$ $(\alpha, \beta=0,123)$. Here $T_{\alpha \beta}$ is the $4 \mathrm{D}$ energy-momentum tensor, which can always be constructed from the $5 \mathrm{D}$ geometry ${ }^{1,2}$ and whose form is now well known. ${ }^{7-9}$ For the present case, $R_{\alpha \beta}$ is given by (3), so the induced energy-momentum tensor is 


$$
T_{\alpha \beta}=R_{\alpha \beta}-\frac{1}{2} g_{\alpha \beta} R=6 \Phi^{-2}\left(\Phi_{\alpha} \Phi_{\beta}-\frac{1}{2} g_{\alpha \beta} \Phi^{\mu} \Phi_{\mu}\right) .
$$

Using (7) and (27), we find that the non-vanishing components of this in mixed form are

$$
T_{0}^{0}=-T_{1}^{1}=-T_{2}^{2}=-T_{3}^{3}=3 \lambda^{2} B^{-1} .
$$

This as expected represents a static source, which we can model as a perfect fluid with

$$
T_{\alpha \beta}=(\rho+p) u_{\alpha} u_{\beta}-p g_{\alpha \beta} .
$$

Here $\rho$ is the density, $p$ is the pressure and the 4 -velocity is $u^{\alpha}=\left(u^{0}, 0,0,0\right)$. Combining (29) and (30), we see that the source has

$$
\rho=p=3 \lambda^{2} B^{-1} .
$$

This is the stiff equation of state in which the speed of sound waves approaches the speed of light, and has been applied in previous studies ${ }^{14,16,21}$ to astrophysical situations such as collapsed stars and proto-galactic fluctuations.

\section{B. TWO-FLUID SOLUTION WITH RADIATION AND HEAT FLOW}

The second solution we look at is obtained by splitting off the whole of the first part of (26). The $4 \mathrm{D}$ metric is then

$$
d \tilde{s}^{2}=\tilde{g}_{\alpha \beta} d x^{\alpha} d x^{\beta}=e^{2 \lambda t}\left[B d t^{2}-A d r^{2}-r^{2} d \Omega^{2}\right],
$$

and is time-dependent. We therefore expect that the source necessary to balance Einstein's equations will also be time-dependent. It should be noted that this property cannot in general be removed by a coordinate transformation based on Birkhoff'sss theorem, because the 4D metric (32) will have a source constructed from the 5D geometry that will in general not be vacuum; and because the $4 \mathrm{D}$ metric (32) is part of a 5D metric (26) and it is known that Birkhoff's theorem in its standard form breaks down in the transition from $4 \mathrm{D}$ to $5 \mathrm{D},{ }^{24,25}$ as evidenced by the existence of both static

and time-dependent soliton solutions. ${ }^{24,26}$ To investigate the time-dependence of (32), let us make the coordinate transformation

$$
e^{\lambda t}=1-\lambda \tilde{t}
$$

This brings (32) into the form 


$$
d \tilde{s}^{2}=\tilde{g}_{\alpha \beta} d \tilde{x}^{\alpha} d \tilde{x}^{\beta}=B d \tilde{t}^{2}-(1-\lambda \tilde{t})^{2}\left(A d r^{2}+r^{2} d \Omega^{2}\right) \quad .
$$

We can use this with $\phi \equiv \Phi^{-2}=e^{-2 \lambda t}=(1-\lambda \tilde{t})^{-2}$ to evaluate the induced matter properties using the standard technique. ${ }^{7-9}$ We note that

$$
\begin{aligned}
\tilde{R}_{\alpha \beta} & =\phi^{-1 \widetilde{\phi_{\alpha ; \beta}}} \\
\tilde{R} & =\phi^{-1 \widetilde{\phi_{; \alpha}^{\alpha}}}=0,
\end{aligned}
$$

which can be used to form the induced energy-momentum tensor:

$$
\tilde{T}_{\alpha \beta}=\tilde{R}_{\alpha \beta}-\frac{1}{2} \tilde{g}_{\alpha \beta} \tilde{R}=\phi^{-1} \widetilde{\phi_{\alpha ; \beta}}
$$

The non-vanishing components of this in mixed form are

$$
\begin{aligned}
& \tilde{T}_{0}^{0}=6 \lambda^{2} B^{-1}(1-\lambda \tilde{t})^{-2} \\
& \tilde{T}_{1}^{1}=\tilde{T}_{2}^{2}=\tilde{T}_{3}^{3}=-2 \lambda^{2} B^{-1}(1-\lambda \tilde{t})^{-2} \\
& \tilde{T}_{0}^{1}=g_{00} g^{11} \tilde{T}_{1}^{0}=\lambda A^{-1} B^{-1} B^{\prime}(1-\lambda \tilde{t})^{-3} .
\end{aligned}
$$

We see from (38) that the density is inhomogeneous via $B=B(r)$ and is timedependent, as is the pressure by (39) though the latter is isotropic. We also see from (40) that there is an off-diagonal component. The latter can be accommodated by introducing a two-fluid model

$$
\tilde{T}_{\alpha \beta}=\tilde{T}_{\alpha \beta}^{(1)}+\tilde{T}_{\alpha \beta}^{(2)}
$$

which as in other work ${ }^{12,16}$ we take to be the sum of a perfect fluid and a radial heat flow:

$$
\begin{aligned}
& \tilde{T}_{\alpha \beta}^{(1)}=(\tilde{\rho}+\tilde{p}) u_{\alpha} u_{\beta}-\tilde{p} \tilde{g}_{\alpha \beta} \\
& \tilde{T}_{\alpha \beta}^{(2)}=q_{\alpha} u_{\beta}+u_{\alpha} q_{\beta} .
\end{aligned}
$$

The heat-flux vector and the 4-velocity must obey the orthogonality condition

$$
q_{\alpha} u^{\alpha}=0
$$

This we satisfy by taking

$$
\begin{array}{ll}
u^{\alpha} & =\left(u^{0}, 0,0,0\right), \quad u^{0}=B^{-1 / 2} \\
q^{\alpha} & =\left(0, q^{1}, 0,0\right),
\end{array}
$$


where in (45) we have used the facts that $r$ is a comoving coordinate and that the 4-velocities are normalized via $g_{\alpha \beta} u^{\alpha} u^{\beta}=B u^{0} u^{0}=1$. Substituting (45), (46) into (42), (43) and these into (41) gives the non-vanishing components of the last in mixed form:

$$
\begin{aligned}
& \tilde{T}_{0}^{0}=\tilde{\rho} \\
& \tilde{T}_{1}^{1}=\tilde{T}_{2}^{2}=\tilde{T}_{3}^{3}=-\tilde{p} \\
& \tilde{T}_{0}^{1}=B^{1 / 2} q^{1} .
\end{aligned}
$$

Comparing these with (38), (39), (40) gives us the density, pressure and heat flow in explicit form:

$$
\begin{aligned}
\tilde{\rho} & =3 \tilde{p}=6 \lambda^{2} B^{-1}(1-\lambda \tilde{t})^{-2} \\
q^{1} & =\lambda A^{-1} B^{-3 / 2} B^{\prime}(1-\lambda \tilde{t})^{-3}
\end{aligned}
$$

The equation of state is that of radiation or ultra-relativistic matter, and has been applied in previous studies ${ }^{16,27}$ to astrophysical situations such as fermion soliton stars and the early universe.

\section{DISCUSSION AND CONCLUSION}

We have taken a 5D metric (1) which contains a 4D sub-metric (6) whose 3D part is spherically symmetric. An expansion in the metric coefficient allows us to recover the Schwarzschild case of general relativity in (17) as the zeroth approximation and a close-to-Schwarzschild case in (25) as the first order approximation to a class of spherically-symmetric solutions. Any solution of the 5D Kaluza-Klein equations in apparent vacuum can be written as a solution of the 4D Einstein equations with matter. Two solutions have then been shown, with matter properties corresponding to those of a stiff fluid (31), and radiation or ultra relativistic particles with heat flow $(50,51)$. These solutions can be applied to astrophysics, but are merely illustrative examples.

In the wider scheme, it is clear that (local) embedding theorems are powerful tools, whether applied to $N<4$ (possibly quantizeable) gravity, ${ }^{2} 4$ D Einstein theory, ${ }^{12}$ $5 \mathrm{D}$ Kaluza-Klein theory, ${ }^{28}$ or 10D superstrings, 11D supergravity and M-theory. ${ }^{3-6}$ Campbell's theorem ensures that any solution in ND can be embedded in a Ricci-flat solution in $(N+1) D .^{1}$ The Schwarzschild solution in $4 \mathrm{D}$ can of course be embedded in a flat space of $N \geq 6 .{ }^{29}$ And any solution in $4 \mathrm{D}$ can be embedded in a flat space of $N \geq 10 .{ }^{30}$ The implications of embedding theorems are diverse. If the aim is to find new solutions of general relativity, the higher-dimensional field equations are often surprisingly tractable, and the method of reduction to $4 \mathrm{D}$ is straightforward. ${ }^{2}$ 
If the aim is to give meaning to higher-dimensional theories, the same method of reduction will inform about physicality in the $4 \mathrm{D}$ world. We should recall that field equations like those of Einstein or Kaluza-Klein allow the dimensionality to be freely chosen, which should be done partly with a view to what physics it is desired to describe and partly with a view to what technical constraints are involved. In the latter regard, it is well known that $N<4$ theories run into problems of formulation which are connected with the degenerate nature of lower-dimensional Riemannian spaces. For $N>4$ theories, problems arise with the physics if the spaces are subject to arbitrary technical constraints., ${ }^{7,31,32}$ Our opinion, therefore, is that future work should be focussed on higher-dimensional, fully covariant theory.

\section{ACKNOWLEDGMENTS}

We thank the Referee for valuable suggestions, and J. Ponce de Leon and W.N. Sajko for comments. This work was supported by NSF of P.R. China and NSERC of Canada. 


\section{Research Bibliography}

${ }^{1}$ J.E. Campbell, A Course of Differential Geometry, Clarendon, Oxford (1926).

${ }^{2}$ S. Rippl, C. Romero, R. Tavakol, Class. Quant. Grav. 12, 2411 (1995); C. Romero, R. Tavakol, R. Zalaletdinov, Gen. Rel. Grav. 28, 365 (1996); J.E. Lidsey, C. Romero, R. Tavakol, S. Rippl, Class. Quant. Grav. 14, 865 (1997).

${ }^{3}$ E. Witten, Nucl. Phys. B 186, 412 (1981); P. Horava, E. Witten, Nucl. Phys. B 460, 506 (1996); 475, 94 (1996).

${ }^{4} \mathrm{P}$. West, Introduction to Supersymmetry and Supergravity, World Scientific, Singapore (1986).

${ }^{5}$ M.B. Green, J.H. Schwarz, E. Witten, Superstring Theory, Cambridge Un. Press, Cambridge (1987).

${ }^{6}$ M.J. Duff, Int. J. Mod. Phys. A 11, 5623 (1996).

${ }^{7}$ P.S. Wesson, Space, Time, Matter, World Scientific, Singapore (1999).

${ }^{8}$ P.S. Wesson, Astrophys. J. 394, 19 (1992).

${ }^{9}$ A. Billyard, P.S. Wesson, Phys. Rev. D 53, 731 (1996).

${ }^{10}$ D. Kalligas, P.S. Wesson, C.W.F. Everitt, Astrophys. J. 439, 548 (1995); H. Liu, J.M. Overduin, Astrophys. J. 538, 386 (2000); J.M. Overduin, Phys. Rev. D 62, 102001 (2000).

${ }^{11}$ B. Mashhoon, H. Liu, P.S. Wesson, Phys. Lett. B 331, 305 (1994); H. Liu, B. Mashhoon, Ann. Phys. (Leipzig) 4, 565 (1995); P.S. Wesson, B. Mashhoon, H. Liu, Mod. Phys. Lett. A 12, 2309 (1997); H. Liu, B. Mashhoon, Phys. Lett. A 272, 26 (2000).

${ }^{12}$ D. Kramer, H. Stephani, M. MacCallum, E. Herlt, Exact Solutions of Einstein's Field Equations, Cambridge Un. Press, Cambridge (1980).

${ }^{13}$ P.C. Vaidya, Nature (London) 171, 260 (1953).

${ }^{14}$ L. Herrera, J. Jimenez, L. Leal, J. Ponce de Leon, M. Esculpi, V. Galina, J. Math. Phys. 25, 3274 (1984).

${ }^{15}$ L. Herrera, J. Ponce de Leon, J. Math. Phys. 26, 2302 (1985); 26, 2847 (1985).

${ }^{16}$ M. Esculpi, L. Herrera, J. Math. Phys. 45, 3341 (1992).

${ }^{17}$ E. Glass, J.P. Krisch, Phys. Rev. D 57, R5945 (1998). 
${ }^{18}$ G. Abolghasem, A.A. Coley, D.J. McManus, J. Math. Phys. 37 , 361 (1996); H. Liu, P.S. Wesson, Gen. Rel. Grav. 30, 509 (1998); P.S. Wesson, H. Liu, Phys. Lett. B 432, 266 (1998).

${ }^{19}$ T. Appelquist, A. Chodos, Phys. Rev. D 28, 772 (1983); H. Liu, P.S. Wesson, Int. J. Mod. Phys. D 7, 737 (1998).

${ }^{20}$ C.W. Misner, D.M. Sharp, Phys. Rev. B 136, 571 (1964); M.P. Podurets, Sov. Astron. (A.J.) 8, 19 (1964); P.S. Wesson, Mon. Not. Roy. Astr. Soc. 197, 157 (1981).

${ }^{21}$ P.S. Wesson, J. Math. Phys. 19, 2283 (1978).

${ }^{22}$ H. Liu, P.S. Wesson, J. Ponce de Leon, J. Math. Phys. 34, 4070 (1993).

${ }^{23}$ H. Liu, P.S. Wesson, Phys. Lett. B 381, 420 (1996); Class. Quant. Grav. 14, 1651 (1997).

${ }^{24}$ D.J. Gross, M.J. Perry, Nucl. Phys. B 226, 29 (1983).

${ }^{25}$ Y.M. Cho, D.H. Park, Gen. Rel. Grav. 23, 741 (1991); P.S. Wesson, in STEP: Testing the Equivalence Principle in Space, European Space Agency, WPP-115, 566 (1996).

${ }^{26}$ R.D. Sorkin, Phys. Rev. Lett. 51, 87 (1983); A. Davidson, D. Owen, Phys. Lett. B 155, 247 (1985); P.S. Wesson, H. Liu, P. Lim, Phys. Lett. B 298, 69 (1993).

${ }^{27}$ T.D. Lee, Phys. Rev. D 35, 3637 (1987); R. Frieberg, T.D. Lee, Y. Pang, Phys. Rev. D 35, 3640 (1987); T.D. Lee, Y. Pang, Phys. Rev. D 35, 3678 (1987); R. Frieberg, T.D. Lee, Y. Pang, Phys. Rev. D 35, 3658 (1987); P.S. Wesson, H. Liu, Astrophys. J. 440, 1 (1995).

${ }^{28}$ T. Kaluza, Sitz, Preuss. Akad. Wiss. 33, 966 (1921); O. Klein, Z. Phys. 37, 895 (1926); J.M. Overduin, P.S. Wesson, Phys. Rep. 283, 303 (1997).

${ }^{29}$ F.R. Tangherlini, Nuovo Cimento 27 (Ser. 10), 636 (1963).

${ }^{30}$ L.P. Eisenhart, Riemannian Geometry, Princeton Un. Press, Princeton (1949).

${ }^{31}$ N. Arkani-Hamed, S. Dimopoulos, G. Dvali, Phys. Lett. B 429, 263 (1998); Phys. Rev. D 59, 086004 (1999); I. Antoniadis, N. Arkani-Hamed, S. Dimopoulos, G. Dvali, Phys. Lett. B 436, 257 (1998).

${ }^{32}$ L. Randall, R. Sundrum, Phys. Rev. Lett. 83, 3370 (1999); 83, 4690 (1999). 